\title{
Presencia de Integrones Clase 1 en Aislamientos de Staphylococcus epidermidis de las unidades de neonatología del Instituto Materno Infantil de Bogotá
}

\author{
Gladys Pinilla M.Sc ${ }^{1} .$, Liliana Muñoz M.Sc. ${ }^{1}$, Eva Amanda Gallego M.Sc. ${ }^{1}$, \\ Bibiana Chavarro B. ${ }^{1}$, Jennifer Fandiño ${ }^{1}$ \\ Grupo Rema: Relaciones microbianas y epidemiológicas aplicadas al laboratorio clínico y molecular. Programa de Bacteriología y \\ Laboratorio Clínico, Universidad Colegio Mayor de Cundinamarca. \\ ${ }^{1}$ Universidad Colegio Mayor de Cundinamarca \\ Correspondencia: gpinillab@unicolmayor.edu.co
}

Recibido: 25-09-06 / Aceptado: 27-10-06

\section{Resumen}

La multiresistencia a los antibióticos por bacterias Gram positivas, especialmente el Staphylococcus epidermidis, constituye un problema creciente de salud pública en las unidades neonatales. Uno de los mecanismos de resistencia bacteriana recientemente descrito es la presencia de integrones y cassettes genéticos. Existen más de 9 clases de integrones, siendo el de clase 1 el que con mayor frecuencia se encuentra implicado en cepas causantes de infección hospitalaria.

En este estudio, se determinó la presencia de integrones clase 1 en Staphylococcus epidermidis, provenientes de hemocultivos y puntas de catéter, que causaron infección nosocomial en las unidades neonatales del Instituto Materno Infantil de Bogotá, Colombia. De las 46 cepas estudiadas, se detectó la presencia del gen intI 1 en 21 de los aislamientos (45.7\%). Este hallazgo, pionero en cepas bacterianas aisladas en neonatos, sugiere la necesidad de buscar cassettes genéticos que confieren resistencia a los diferentes antimicrobianos, para definir esquemas de tratamiento de infecciones nosocomiales causadas por estos microorganismos.

Palabras clave: antimicrobianos, cassettes genéticos, genoma bacteriano, integrones, neonato, plásmidos.

\begin{abstract}
Multiresistance to antimicrobial against Gram-positive bacteria, especially Staphylococcus epidermidis is a progressive problem in newborn-intensive care units. One of the recently described mechanisms of bacterial ressistance is the presence of integrons and gene cassettes. There are more than 9 types of integrons, out of which class 1 integron is the most frequently associated with strains causing hospital infections.

In this study, presence of class 1 integrons in Staphylococcus epidermis was determined as coming from hemocultures and catheter points which caused nasocomial infection in newborn intensive care units of Instituto Materno Infantil in Bogotá, Colombia. From the 46 studied strains, intll gene was detected in 21 of them (45.7\%). This finding, pioneer in bacterial strains isolated in newborns, suggests the necessity to look
\end{abstract}


for gene cassette which give resistance to the different anti microbial entities to define nosocomial infection treatment schemes caused by these microorganisms.

This is the first time mechanism of resistance to aminoglycoside and other antimicrobials in Colombian Gram positive strains is studied. We suggest that it is important to carry out assays with gene cassette for different antibiotics of nosocomial infection to provide a better treatment selection.

Key words: antimicrobial, gene cassette, genome bacterial, integrons, newborn, plasmids.

\section{Introducción}

El Staphylococcus epidermidis es el germen más frecuente aislado en las unidades de neonatología, ha sido reconocido en las ultimas décadas como el mayor causante de las infecciones nosocomiales en recién nacidos, presentando un alto índice de morbi-mortalidad y multiresistencia a los antibióticos $(1,2)$.

Dentro de los mecanismos de resistencia a los antimicrobianos por transferencia horizontal de genes, se encuentra una nueva familia de elementos de ADN móviles denominados integrones; se localizan en el genoma bacteriano, en plasmidos o en transposones ( 3 , $4,5,6)$.

La estructura básica del integron consiste en dos segmentos conservados en los extremos 5' y 3', separados por un segmento de longitud variable, donde se insertan cassettes genéticos con uno o más genes que confieren resistencia a los antibióticos, mediante un sistema de recombinación sitio-especifica $(11,12)$. En el extremo 5' se encuentra el gen intI, que codifica para una proteína con actividad de recombinasa o integrasa; contiguo a este, se localiza la secuencia attI, sitio de recombinación especifica donde se integran cassettes genéticos separados por pequeñas regiones intergénicas de aproximadamente $10 \mathrm{pb}$. Entre intI y
attI están los promotores $\mathrm{P}_{\mathrm{c}}$ y $\mathrm{P}_{\mathrm{i}}$, que codifican para la expresión de cassettes génicos y para la expresión de intI1 respectivamente (Figura 1) $(12,13)$.

La enzima IntI permite la interacción entre attl y el sitio $a t t C$, el cual une los extremos del cassette genético y facilita la integración en la zona variable del integron (14). En el extremo 3' del integron clase 1, se han descrito varios genes que confieren resistencia a compuestos de amonio cuaternario ( $q a c E \ddot{A} 1$ ), que hacen parte de algunos antisépticos y desinfectantes; así como de genes que determinan resistencia a las sulfonamidas (sul1) y el gen orf 5 cuya función se desconoce hasta el momento (15).

El integron clase 1 es el más estudiado en bacterias Gram negativas, mientras que su presencia en bacterias Gram positivas es menos conocida $(7,8,9,10)$. En este estudio se determinó la presencia de integrones clase 1, en aislamientos de $S$. epidermidis provenientes de hemocultivos y cultivos de punta de catéter de las unidades de neonatología del Instituto Materno Infantil de Bogotá (IMI). La determinación de la presencia de integrones y su relación con cassettes genéticos de resistencia antimicrobiana, especialmente para aminoglucosidos y betalactamicos, utilizados en las

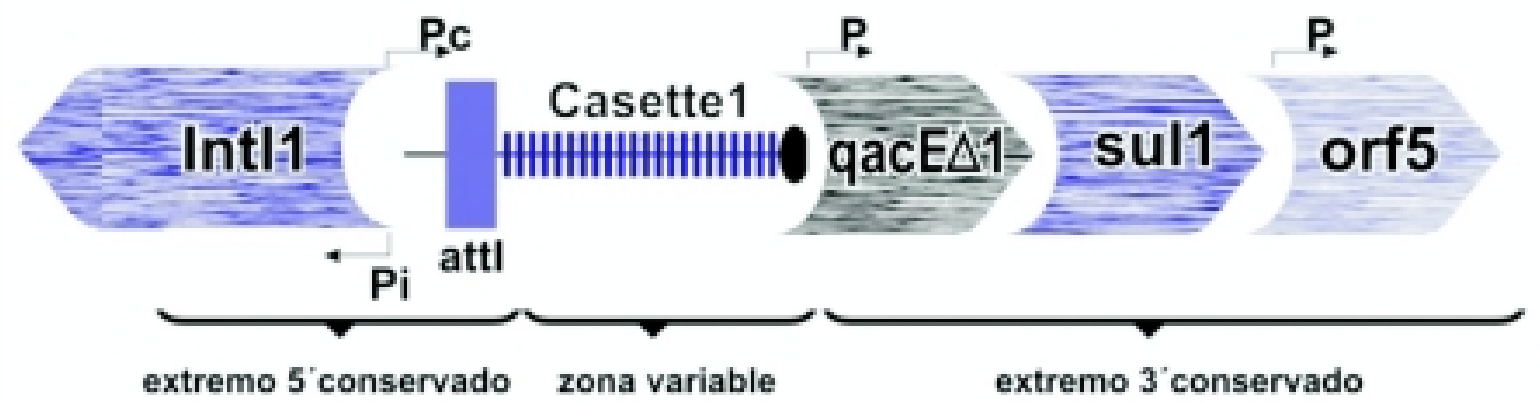

Figura 1. Estructura del integron clase 1. 
unidades de neonatología, permitirá la intervención más eficaz en el tratamiento de infecciones nosocomiales causadas por S. epidermidis.

\section{Materiales y Métodos}

\section{Aislamientos}

Se estudiaron 46 cepas de $S$. epidermidis aisladas a partir de hemocultivos y cultivos de puntas de catéter provenientes de 38 recién nacidos admitidos en las unidades de neonatología del IMI durante el periodo comprendido entre el 21 de noviembre de 2003 y el 20 de Mayo de 2004. La identificación de las especies se realizó por el método MicroScan (Roche Biocare, Canadá).

\section{Análisis por Bioinformática}

La búsqueda del gen de la integrasa tipo I (intll), se realizó a partir de secuencias reportadas en la base de datos del NCBI (Nacional Center Biotechnology Information), pertenecientes a los géneros Klebsiella, Salmonella, Serratia, Acinetobacter, y Citrobacter. Dichas secuencias se utilizaron para la construcción de una secuencia consenso mediante el programa Multalin (disponible en red). A partir de esta secuencia consenso se seleccionó la región más conservada para el diseño de oligonucleotidos empleando el programa Primer3 (20).

\section{Amplificación del gen intI1 por reacción en cadena de la polimerasa}

La extracción de DNA se realizó a partir de un cultivo puro, siguiendo el protocolo del fabricante (Kit Wizard $\mathbb{}$ )
Genomic DNA Purification A1123, Promega, USA). La amplificación del gen intl1 se realizó por PCR utilizando: oligonucleótidos $1.0 \mu \mathrm{M}$, (sentido: C GA A C C GA A C A G GC T TATGT; antisentido: AGCACCTTGCCGTAGAAGA) GoTaq polimerasa $1 \mathrm{U}$, mezcla de dNTPs $200 \mu \mathrm{M}$, DNA $150 \mathrm{ng} / \mu \mathrm{l}$ para un volumen final de $20 \mu 1$. Las condiciones de amplificación fueron: desnaturalización inicial a $94^{\circ} \mathrm{C}$ por 4 minutos, seguida de 30 ciclos de desnaturalización a $94^{\circ} \mathrm{C}$ por 30 segundos, anillamiento a $53^{\circ} \mathrm{C}$ por 45 segundos, extensión a $72^{\circ} \mathrm{C}$ por 45 segundos y una extensión final a $72^{\circ} \mathrm{C}$ por 7 minutos. La detección del producto de amplificación de aproximadamente $188 \mathrm{pb}$, se realizó mediante electroforesis en gel de agarosa al $2 \%$, seguido de tinción con bromuro de etidio. Como cepa control se utilizó un aislamiento clínico de Salmonella typhi, portador del gen intl1 (Gen Bank EF051039).

\section{Resultados}

Durante el periodo comprendido entre el 21 de noviembre de 2003 y el 20 de Mayo de 2004 se aislaron 46 cepas de $S$. epidermidis, a partir de hemocultivos y cultivos de puntas de catéter provenientes de 38 recién nacidos de la Unidad de Neonatología del IMI. De 46 aislamientos, 21 (45.7\%) fueron positivos para la presencia del gen intll y 25 (54.3\%) fueron negativos. El resultado positivo se evidenció por la presencia de una banda correspondiente a un tamaño de producto de PCR de aproximadamente 188 pb (Figura 2). La tabla 1 muestra la distribución de frecuencias de las cepas provenientes de hemocultivos y punta de catéter relacionadas con la presencia o ausencia del integron clase I.

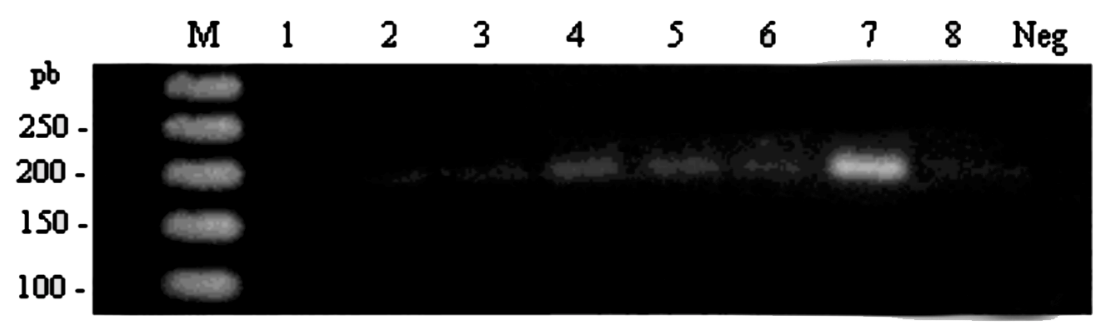

Figura 2. Amplificación del gen intl 1. M: Marcador de peso molecular de 50 pb.; líneas. 2 - 6 y 8 cepas de ECN positivas para el gen intl1 correspondientes a un tamaño de 188 pb. Línea 7 Cepa control Salmonella tiphy (aislamiento clínico, portador del gen intl1). Neg: Control negativo. 
Tabla 1. Frecuencia de presentación del integron clase I (gen intl1) en muestras de hemocultivo y puntas de catéter.

\begin{tabular}{lccccc}
\hline TIPO DE MUESTRA & $\begin{array}{c}\text { NO. DE } \\
\text { MUESTRAS }\end{array}$ & \multicolumn{2}{c}{$\begin{array}{c}\text { PRESENCIA DE } \\
\text { INTEGRON }\end{array}$} & \multicolumn{2}{c}{$\begin{array}{c}\text { AUSENGIA DEL } \\
\text { INTEGRON }\end{array}$} \\
\hline Hemocultivos & & $\mathrm{n}$ & $\%$ & $\mathrm{n}$ & $\%$ \\
Catéteres & 36 & 18 & 50 & 18 & 50 \\
\hline Total & 10 & 3 & 30 & 7 & 70 \\
\hline
\end{tabular}

\section{Discusión}

Entre las bacterias Gram positivas, los Estafilococos coagulasa negativa (ECN) y particularmente el $S$. epidermidis, muestra una alta prevalencia $(>70 \%)$ en las infecciones neonatales en el mundo, y constituye uno de los principales patógenos aislados en las Unidades de Neonatología, presentando multiresistencia a la terapia antimicrobiana $(16,17,18)$. Los resultados del presente estudio muestran la presencia de integrones clase 1 en aislamientos clínicos de $S$. epidermidis, obtenidos de hemocultivos y cultivos de puntas de catéter de las unidades de neonatología del IMI, durante el periodo comprendido entre el 21 de noviembre de 2003 y el 20 de Mayo de 2004.

Se amplificó el gen intll en el genoma bacteriano, que corresponde a un integron de resistencia clase 1, siendo este el más estudiado, debido a que se presenta con mayor frecuencia en aislamientos clínicos. De los 46 aislamientos de $S$. epidermidis, 21 (45.7\%) fueron positivos para el gen intI1. La presencia del integron clase 1 sólo había sido reportada en ECN, en un estudio realizado en China, donde la frecuencia fue del 100\%; sin embargo, dicho estudio no fue realizado en neonatos (7).

Los integrones se han reconocido como elementos móviles importantes en la evolución del genoma bacteriano y se consideran como uno de los mecanismos genéticos responsables de la resistencia a los antibióticos de amplio espectro usados en el ámbito hospitalario, en aislamientos clínicos de bacterias Gram negativas. Recientemente se encontró la primera evidencia de integron clase 1 en microorganismos Gram positivos asociados a infección nosocomial (19).

Los integrones clase 1 podrían ser mediadores de resistencia a diferentes antibióticos en aislamientos clínicos de bacterias Gram positivas, como $S$. aureus, $S$. epidermidis, S. haemolyticus, Estreptococcus sp. entre otros, que presentaron resistencia a diferentes antimicrobianos como Clindamicina, gentamicina, cloranfenicol, ciprofloxacina, eritromicina, tetraciclina (7).

Los ECN han sido subestimados como agente causal de infección. La presencia del integron clase 1 en el $45.7 \%$ de los aislamientos analizados, muestra la importancia de la determinación de la presencia de otras clases de integrones (clases 2 y 3 ), así como el estudio de los cassettes genéticos que median resistencia a los aminoglucósidos, utilizados en el tratamiento de infecciones en las unidades de neonatología. La unión entre los hallazgos clínicos y moleculares contribuirá en la definición de esquemas de tratamiento adecuados, así como en la implementación de programas para la prevención y control de patógenos que son considerados por muchos clínicos como simples contaminantes.

\section{Referencias}

1. Ardic N, Sareyyupoglu B, Ozyurt M, Haznedaroglu T, Ilga U Investigation of aminoglycoside modifying enzyme genes in methicillin-resistant staphylococci: Microbiol Res. 2006;161:49-54.

2. Cifuentes Y, Ruiz A, Leal A, Muñoz L, Herrera M, Jimenez L. Perfil microbiológico en asilamientos en Unidades Neonatales en un hospital de tercer nivel de Bogotá, Colombia. Rev. Salud Pública 2005; 7:191-205.

3. Stockes $\mathrm{H}$ qnd Hall R. A novel family 9of potencially mobile DNA elements encoding site-specific gene integration finctions: integrons. Molec. Microbial. 1989; 3:1669-1683.

4. Guillemont TrwB, the coupling protein involved in DNA transport during bacterial conjugation, is a DNA-dependent ATPase. Biochemistry 2005; 102:8156-8161.

5. Gonzalez R, Mella S, Zemelman R, Bello H, Dominguez M. Integrones y cassettes genéticos de resistencia: estructura $\mathrm{y}$ rol frente a los antibacterianos. Rev. Méd. Chile, 2004; 132:619-626.

6. Bennett, P and Howe T. Bacterial and bacteriophage genetics. In Topley \& Wilson's Microbiology and MicrobialInfections, 1998Vol. 2, Systematic Bacteriology, 9th edn, (Collier, L.,Balows, A. \& Sussman, M., Eds), pp. 231-294. Arnold, London. 
7. Shi L, Zheng M, Xiao Z, Asakura M, Su J, Li L, Yamasaki S. Unnoticed spread of class 1 integrons in gram-positive clinical strains isolated in Guangzhou, China. Microbiol Immunol. 2006; 50:463-467.

8. Nandi S, Maurer JJ, Hofacre C, Summers AO. Gram-positive bacteria are a major reservoir of Class 1 antibiotic resistance integrons in poultry litter. Proc Natl Acad Sci U. S A. 2004; 101:7118-7122.

9. Nesvera J, Hochmannova J, and Patek M. An integron of class 1 is present on the plasmid Pcg4 front Gram positive bacterium Corynebacterium glutamicum FMS Microb. Lett. 1998; 169:391-395.

10. Tauch A, Gotker S, Puhler A, Kalinowski J, and Thierbach G. The 27.8-Kb R-Plasmid pTET3 from Corynebacterium glutamicum encodes the aminoglycoside adenyltransferase gne cassette aadA9 and the regulated tetracycline efflux system Tet 33 flanked by active copies of the widespread insertion sequence IS6100. Plasmid 2002; 48:117-129.

11. Collis CM, Hall RM. Expression of antibiotic resistance genes in the integrated cassettes of integrons. Antimicrob Agents Chemother. 1995; 39:155-162.

12. Gonzalez G, Mella S, Zemelman R, Bello H and Dominguez Y. Integrones y cassettes genéticos de resistencia: estructura y rol frente a los antibacterianos. Rev. Méd. Chile, 2004, 132:619-626.

13. Stokes HW, O'Gorman DB, Recchia GD, Parsekhian M, Hall RM. Structure and function of 59-base element recombination sites associated with mobile gene cassettes. Mol Microbiol. 1997; 26:731-45.
14. Martinez-Freijo P, Fluid A, Schnitmitz F, Greck V, Verhoef J and Jones M. Class I integrons in Gram-negative isolates from different European hospital and association with decreased susceptibility to multiple antibiotics compounds. J. Antimicrob Cmeoter. 1998; 42:689-696.

15. Fluit A and Schmitz F.-J. Resistance integrons and superintegrons, Clin. Microbio. Infec. 2004; 10:272-288.

16. Pessoa- Silva C, Miyasaki C, de Almeida M, Kopelman B, Raggio R, Wey S. Neonatal late-onset bloodstream infection: attributable mortality, excess of length of stay and risk factors. Eur. J Epidemiol. 2002; 39:1034-1039.

17. Cunha Ma de L, Lopes C, Rugolo L, Chalita L. clinical significance of coagulase-negative staphylococci isolated from neonates. J Pediatr (Rio J) 2002; 78:279-288.

18. Cunha Mde L, Rugolo LM, Lopes CA. Study of virulence factors in coagulase-negative staphylococci isolated from newborns. Mem Inst Oswaldo Cruz. 2006; 101:661-668.

19. Rowe-Magnus DA, Mazel D. The role of integrons in antibiotic resistance gene capture. Int J Med Microbiol. 2002; 292:115-125.

20. Steve Rozen, Helen J. Skaletsky. 2000. Primer3 on the WWW for general users and for biologist programmers. In: Krawetz S, Misener S (eds) Bioinformatics Methods and Protocols: Methods in Molecular Biology. Humana Press, Totowa, NJ, pp 365-386. Source code available at http://fokker.wi.mit.edu/ primer3/. 